\title{
332 - Electroconvulsive therapy in older adults with major depression was not associated with cognitive decline during a 15-year follow-up
}

AUTHORS: Kerstin Johansson, Karolina Thömkvist, Ingmar Skoog and Sacuiu SF* (*presenter)

OBJECTIVE: To determine the effects of electroconvulsive therapy (ECT) in major depression in relation to the development of dementia during long-term follow-up.

METHOD: In an observational clinical prospective study of consecutive patients 70 years and older diagnosed with major depression at baseline 2000-2004 ( $n=1090)$, who were free of dementia and received antidepressant treatment, with or without ECT, we sought to determine if cognitive decline (mild cognitive impairment and dementia) during 15-year follow-up was associated with receiving ECT at baseline. The control group was selected among the participants in the Gothenburg H70 Birth Cohort Studies matched by age group and sex 1:1.

RESULTS: Among patients with affective syndromes 7\% received ECT. During follow-up, 157 patients were diagnosed with dementia, equal proportions among those who received ECT (14.5\%) and those who did not receive ECT (14.5\%). The relation between ECT and cognitive decline remained nonsignificant irrespective antidepressive medication or presence of mild cognitive impairment at baseline.

CONCLUSION: Preliminary results indicate that ECT was not associated with the development of cognitive decline in the long-term in a hospital-based cohort of $70+$ year-olds. The results remain to verify against controls from a representative community sample. 